www.jmscr.igmpublication.org

Impact Factor 5.84

Index Copernicus Value: 71.58

ISSN (e)-2347-176x ISSN (p) 2455-0450

crossref DOI:_https://dx.doi.org/10.18535/jmscr/v5i11.32

Journal Of Medical Science And Clinical Research

\title{
A Study of Prevalence of Non Diabetic Renal Disease in Patients with Diabetic Nephropathy
}

\author{
Authors

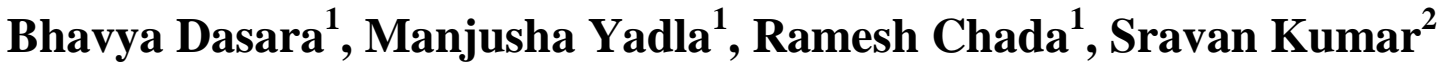 \\ ${ }^{1}$ Department of Nephrology, Gandhi Hospital, Hyderabad \\ ${ }^{2}$ Department of Pathology, Gandhi Hospital, Hyderabad \\ *Corresponding Author \\ Bhavya Dasara \\ Email: bhavyad3@gmail.com
}

\section{Introduction}

Diabetic nephropathy is one of the leading cause of ESRD .It is also one of the most significant long term complications in terms of morbidity and mortality in patients with diabetes. Furthermore, prognosis in such patients is very poor compared with patients with ESRD due to other renal diseases. Diabetic nephropathy (DN) is suspected based on the presence of proteinuria, decline in GFR and elevation of blood pressure. Renal biopsy is not routinely performed in diabetic nephropathy patients. Recently, attention has been drawn to atypical presentation of diabetic and non diabetic glomerular diseases. Renal biopsy in proteinuric patients with diabetes mellitus is to confirm/exclude non-diabetic renal disease (NDRD). There are no standardized criteria for renal biopsy in $\mathrm{DN}$, therefore doing a renal biopsy for patients with DN is the based on decision of the the treating nephrologist.

The prevalence of Non-diabetic renal diseases (NDRD) from various studies ranged from 23$54 \%$ in type 2 DM patients. Identification of Nondiabetic renal diseases is important as diabetic nephropathy is difficult to reverse in comparsion to NDRD which when treated adequately can be reversed. Therefore, kidney biopsy may become a useful diagnostic option among proteinuric patients with diabetes mellitus.

In 2010 Research Committee of the Renal Pathology Society (RPS) developed a consensus classification combining type 1 and type 2 diabetic nephropathies by Tervaert et al in this classification, diabetic nephropathy is divided in to four hierarchical glomerular lesions with separate evaluation for interstitial and vascular lesions. However the clinical utility of this pathological classification for predicting outcomes is not established. This study aimed to evaluate the relationship between histological changes and clinical parameters in diabetic patients with renal dysfunction.

\section{Materials and Methods}

\section{Aim of the study}

- To study the prevalence of non diabetic renal disease in patients with diabetic nephropathy. 
- To study histopathological correlation of diabetic nephropathy with clinical parameters.

\section{Inclusion criteria}

- All diabetic patients with renal dysfunction admitted in the wards of Nephrology Gandhi hospital, who underwent renal biopsy during December 2013 to December 2015 were included.

\section{Exclusion criteria}

- Acute precipitating event for renal dysfunction

- Patients with bilateral contracted kidneys.

- Patients with contraindications for renal biopsy

- Patients unwilling for renal biopsy

The prospective study was carried out from December 2013 to December 2015 in the Department of Nephrology, Gandhi Hospital.

Of 139 diabetic patients with presumed diabetic kidney disease admitted, 43 patients have contracted kidneys, 8 patients have acute precipitating event and 26 patients who were not willing for biopsy were excluded from the study. 62 patients were biopsied and their histopathology studied.

Informed consent was obtained from each patient before biopsy. Diagnosis of diabetes was made using ADA criteria for diagnosis of Diabetes ${ }^{6}$. Detailed history with regards to type of diabetes , duration, treatment for diabetes, details of renal symptoms, micro and macro vascular complications and clinical examination was done. Fundus examination was performed by single ophthalmologist. Further evaluation viz, renal profile (blood urea, serum creatinine, serum electrolytes, complete urine examination, $24 \mathrm{hr}$ urinary protein) was done. eGFR was calculated by the MDRD formula in adults and Schwartz formula for children. RBS, FBS, PLBS, complete hemogram, liver function tests, CT, BT, PT, INR, and appropriate imaging and radiological investigations were done. Ultrasonographic examination was done to assess the renal size. Renal biopsy was performed in 62 patients after stabilization under ultrasound guidance with a biopsy gun (BARD gun $18 \mathrm{G}, 22 \mathrm{~mm}$, cutting edge). 2 samples were collected in all, samples were analyzed under light microscope and immunoflourescence by a single pathologist. All universal precautions were executed during the biopsy. Processed tissue was stained with hematoxylin and eosin ( $\mathrm{H} \& \mathrm{E})$, PAS, silver methenamine, and Masson trichrome for light microscopy. Tissue for IF was stained with fluorescent labeled antisera to $\mathrm{IgG}, \operatorname{IgM}, \operatorname{IgA}, \mathrm{C} 3$, CIq, and Fibrinogen. The intensity was semiquantatively scored, as 0 for negative, $1+$ for present, 2+ for definite and 3+ for strongly positive. Vital parameters were monitored in the immediate post biopsy period. Renal lesions in diabetic nephropathy were classified according to "Pathologic Classification of Diabetic Nephropathy" by - Thijs W. Cohen Tervaer et al, Renal pathology society. This classification scheme is based on glomerular lesions.

Patients with severe renal insufficiency were supported with either intermittent peritoneal dialysis (IPD) or hemodialysis (HD). IPD was due using rigid catheter and using $1.7 \%$ PD solution with as exchange volume of $30-50 \mathrm{ml} / \mathrm{kg}$ for children and 1.5-2 liter for adults. HD was performed using 1.2 square meter hollow fibre for the duration of 4 hours with either femoral or jugular catheter of appropriate size as vascular access.

In all cases renal histopathology was analyzed in correlation with age, sex, duration of DM, Proteinuria, Fundus examination, RFT/eGFR, associated hypertension, glycemic control and need for renal replacement therapy. Fundus examination was done by single ophthalmologist by direct ophthalmoscopy in all cases and 78D/90D Slit lamp biomicroscopy where ever indicated.

Statistical analysis was performed by utilizing SPSS software. Initially frequency tables were made to estimate the frequency \& percentage of each parameter analyzed. Descriptive statistics 


\section{JMSCR Vol||05||Issue||11||Page 30002-30014||November}

were expressed in terms of minimum, maximum, mean \& standard deviation. Logistic regression was used for the prediction of occurrence of an event. The probability of association between two discrete attributes was made by chi square test.

\section{Proforma}

$\begin{array}{lrcr}\text { Name } & \text { Age } & \text { Sex } & \text { Occupation } \\ \text { IPNo } & \text { DOA } & \text { DOD } & \end{array}$

Type of diabetes:

Duration of diabetes:

\section{Complaints \& Duration}

H/O Present illness

- H/o oliguria

- H/o polyuria, nocturia

- H/o hematuria/pyuria/gravelluria/ dysuria/frequency /urgency

- H/o obstructive symptoms

- H/o pedal odema/ SOB/

- H/o Azotemia

- H/o blurring of vision/retinal surgeries/laser photocoagulation

- H/o parasthesias

- $\mathrm{H} / \mathrm{o}$ recent $\mathrm{GE} /$ precipitaing factors

- H/o usage of nephrotoxic drugs/herbal medicine intake

Past History

- HTN/CKD/CAD/TB/asthma/epilepsy

Family History

-DM/HTN/CKD/

Menstrual \& Obstretic H/O

Drug H/O- ?OHA/ ?INSULIN

General Examination

- Pallor/Icterus/Cyanosis/Clubbing/Lymphadenopathy/Edema

-Height cms Weight kgs BMI

Vital data

Temp Pulse

RR

BP

Systemic examination

P/A-

Fundus

Provisional diagnosis

Investigations

$\mathrm{CBP}: \mathrm{Hb} \%$

CUE: Pr:

RBC:

24 hr Urinary prot

/day Sug:

RBS :

$\mathrm{mg} / \mathrm{dl}$

FBS: mg/dl

PLBS :

$\mathrm{mg} / \mathrm{dl}$

HbA1C:

Bl. Urea:

$\mathrm{mg} / \mathrm{dl}$

Sr Creat :

$\mathrm{mg} / \mathrm{dl}$ 


$\begin{array}{llll}\text { Sr Na+ : } & \text { meq/L } & \mathrm{K}+: & \mathrm{meq} / \mathrm{L} \\ \text { Sr proteins: Total- } & \text { G/dl } & \text { Sr albumin : } & \text { G/dl } \\ \text { Sr Ca++ : } & \text { mg/dl } & \text { Sr Bilirubin-: } & \mathrm{mg} / \mathrm{dl}\end{array}$

CT, BT:

USG abdomen: Rt kidney Lt Kidney

Renal biopsy

- LM

- IF

\section{Diagnosis}

\section{Observations}

This prospective study was carried out from Dec 2013 to Dec 2015 in Department of Nephrology, Gandhi hospital. Of 139 diabetic patients with renal dysfunction admitted, 77 patients i.e those patients with contracted kidneys (43), acute precipitating event (8) and patients who are unwilling for biopsy(26) were excluded from the study. A total of 62 diabetic patients with renal dysfunction who underwent renal biopsy were studied. Type1 Diabetes was present in 2 cases and 60 cases had type 2 Diabetes.

Of 62 patients studied 45 patients had Diabetic nephropathy and 17 patients had Non diabetic renal disease.

\section{Age Distribution}

Average age of study population was $49.96 \pm 8.06$ years with youngest patient being 30

Yrs of age and eldest being $70 \mathrm{yrs}$ of age. $70 \%$ of sample are between 41-60 yrs of age.

\begin{tabular}{|l|c|}
\hline AGE IN YEARS & STUDY SAMPLE \\
\hline $0-20$ & 0 \\
\hline $21-40$ & 9 \\
\hline $41-60$ & 49 \\
\hline$>60$ & 4 \\
\hline TOTAL & 62 \\
\hline
\end{tabular}

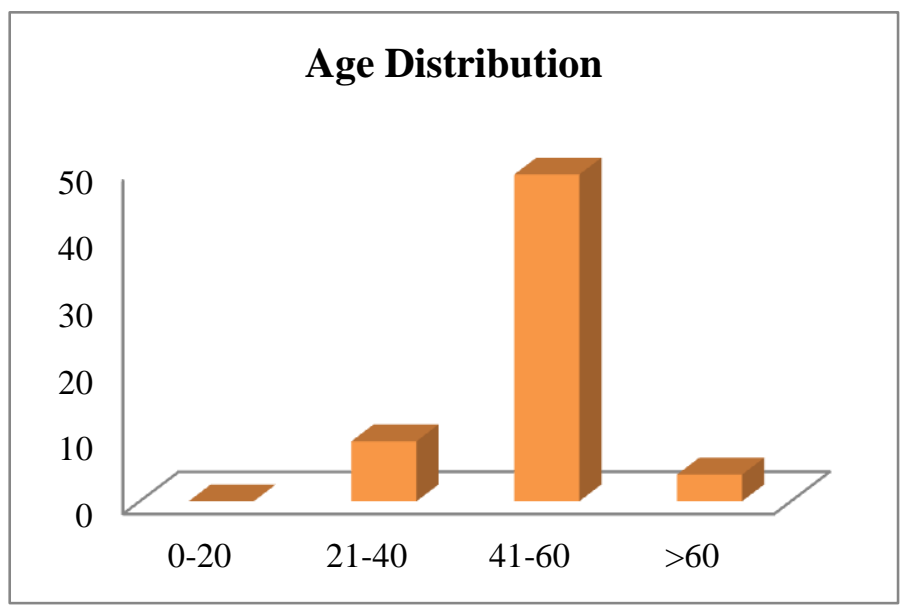

\section{Gender Distribution}

Of the 62 cases 49 were male and 13 were females with a male:female ratio is $3.7: 1$

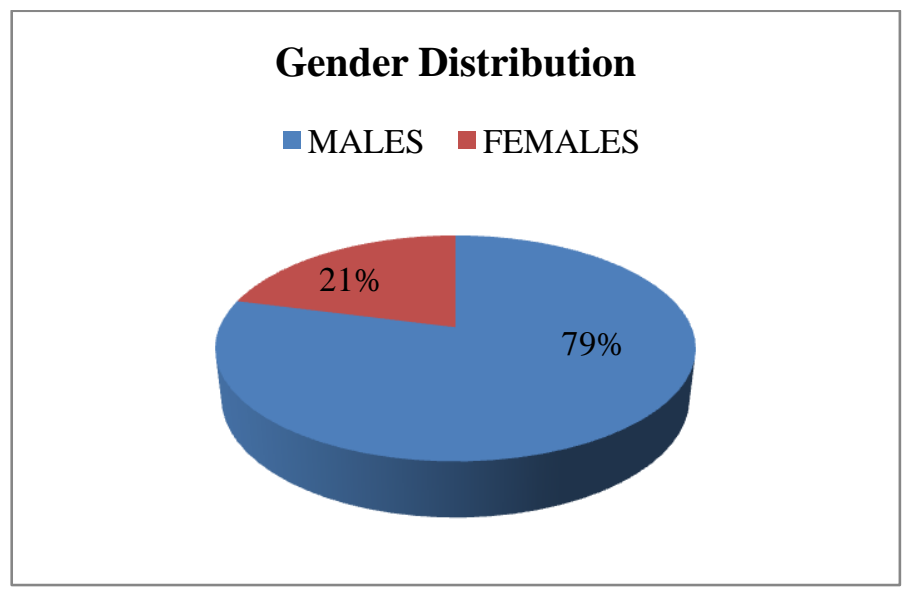

\section{Duration Of Diabetes}

In the present study mean duration of diabetes was $6.95 \pm 4.36$ years in DN cases and $5.69 \pm 4.25$ years in NDRD cases. 


\begin{tabular}{|l|c|c|}
\hline Duration In Years & No Of Cases & Percentage \\
\hline $0-5$ Years & 30 & $48 \%$ \\
\hline 6-10years & 26 & $42 \%$ \\
\hline$>10$ Years & 6 & $10 \%$ \\
\hline Total & 62 & $100 \%$ \\
\hline
\end{tabular}

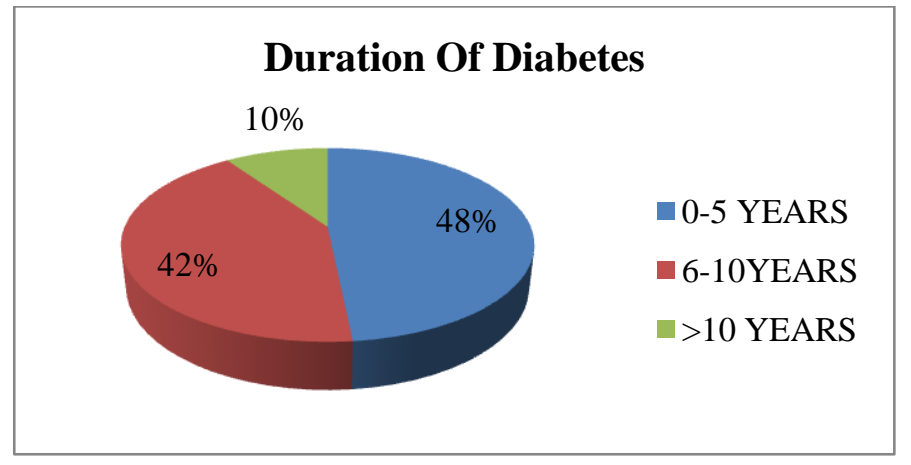

\section{Histopathology}

Of 62 cases studied $\mathbf{4 5}(\mathbf{7 2 . 5 \%})$ cases had Diabetic nephropathy -DN, 17(27.4\%) cases had nondiabetic renal disease-NDRD on histopathology.

\section{Pathological Classification of DN}

\begin{tabular}{|l|c|c|}
\hline CLASS I & 2 & $4 \%$ \\
\hline CLASS IIa & 2 & $4 \%$ \\
\hline CLASS IIb & 5 & $11 \%$ \\
\hline CLASS III & 14 & $31 \%$ \\
\hline CLASS IV & 22 & $49 \%$ \\
\hline TOTAL & 45 & $100 \%$ \\
\hline
\end{tabular}

Histopathogical Classification of diabetic renal disease

Of the 45 cases with diabetic nephropathy on histopathology, most common Class of DN was Class IV observed in 22(49\%) cases, followed by Class III DN observed in 14(31\%) cases, followed by Class IIa(4\%) and Class IIb (11\%), followed by Class I DN observed in 2(4\%) cases

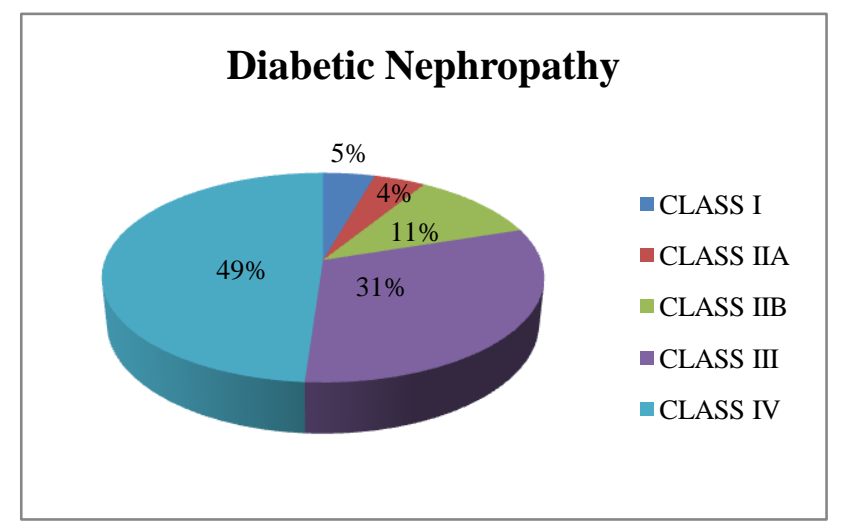

\section{Non Diabetic Renal Disease}

\begin{tabular}{|l|c|}
\hline IgA Nephropathy & 4 \\
\hline FSGS & 3 \\
\hline Membranous N & 1 \\
\hline C3GN & 1 \\
\hline PIGN & 2 \\
\hline HTN Nephropathy & 4 \\
\hline CIN & 1 \\
\hline ATIN & 1 \\
\hline TOTAL & 17 \\
\hline
\end{tabular}

In the present study glomerular lesion were seen in $15(88.3 \%)$ cases and tubulointerstitial lesions were seen in $2(11.7 \%)$ cases. Most common NDRD in present study was IgA Nephropathy seen $4(23.5 \%)$ cases. Cresentric GN was observed in 1 case.

\section{Diabetic Retinopathy}

Of the 62 cases, 32 pts $(51.6 \%)$ had diabetic retinopathy and 30 patients $(48.3 \%)$ did not have diabetic retinopathy.

Of the 32 cases who had diabetic retinopathy 23 cases $(71.8 \%)$ had DN, 9cases (28.1\%) had NDRD. Of 30 cases who did not have diabetic retinopathy 22 cases $(73.3 \%)$ had DN on biopsy.

\begin{tabular}{|l|c|c|}
\hline & DR & NO DR \\
\hline DN & 23 & 22 \\
\hline NDRD & 9 & 8 \\
\hline TOTAL & 32 & 30 \\
\hline
\end{tabular}

Presence or absence of diabetic retinopathy poorly correlated with presence or absence of DN (p value- 0.8 )

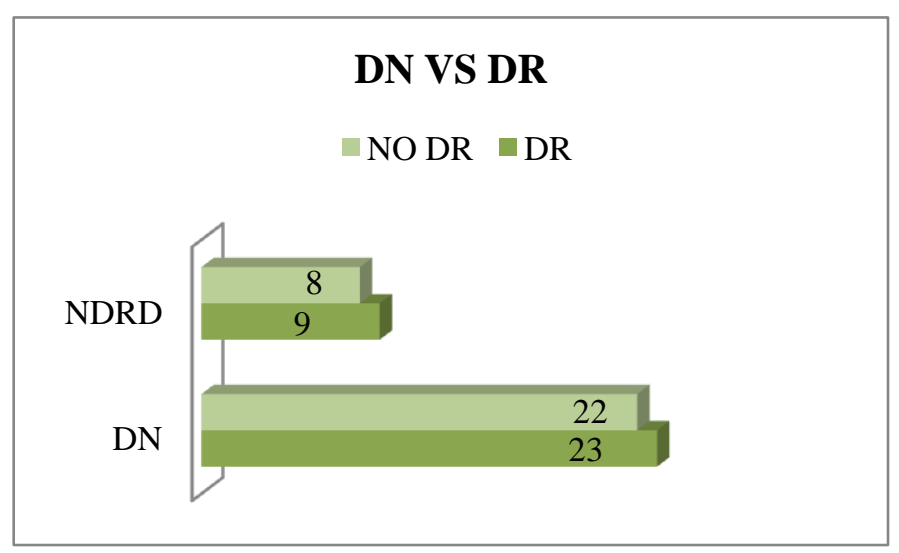


Both cases of Class I DN and Class IIa did not have DR,( $20 \%$ )of cases of Class IIb DN had DR,( $50 \%$ )of Class III DN had DR and (68.1\%) of cases with Class IV DN had DR.

\begin{tabular}{|l|c|c|}
\hline & DR & NO DR \\
\hline CLASS I & 0 & 2 \\
\hline CLASS IIa & 0 & 2 \\
\hline CLASS IIb & 1 & 4 \\
\hline CLASS III & 7 & 7 \\
\hline CLASS IV & 16 & 6 \\
\hline TOTAL & 24 & 21 \\
\hline
\end{tabular}

Prescence of diabetic retinopathy correlated with higher class of diabetic nephropathy (p0.03)

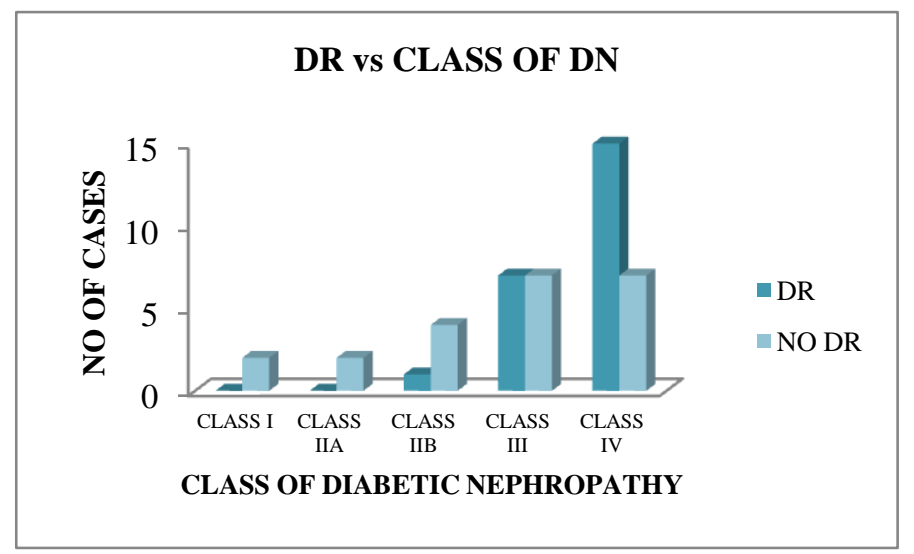

\section{DN \& Hypertension}

Of the 62 cases of diabetic patients with renal dysfunction $46(74.1 \%)$ cases had hypertension. 34 (75\%) hypertensive cases had diabetic nephropathy and 12 (70.5\%) cases have NDRD

\begin{tabular}{|l|c|c|}
\hline NO.OF CASES & HTN & Normotension \\
\hline DN & 34 & 11 \\
\hline NDRD & 12 & 5 \\
\hline TOTAL & 46 & 16 \\
\hline
\end{tabular}

Presence of hypertension didn't predict the presence or absence of DN (p -0.94)

\begin{tabular}{|l|c|c|}
\hline & NORMOTENSIVE & HYPERTENSIVE \\
\hline CLASS I & 2 & 0 \\
\hline CLASS IIa & 1 & 1 \\
\hline CLASS IIb & 1 & 4 \\
\hline CLASS III & 2 & 12 \\
\hline CLASS IV & 5 & 17 \\
\hline
\end{tabular}

Presence of Hypertension didn't correlate with class of Diabetic nephropathy (p value-0.1)

\section{DN Class \& Duration of Diabetes}

Mean duration of diabetes in cases with diabetic nephropathy was $6.9 \pm 4.22$ yrs, Mean duration of DN in NDRD was 5.64 $\pm 4.36 y r s$.

\begin{tabular}{|l|c|c|c|}
\hline & 0-5YEARS & 6-10YEARS & >10YEARS \\
\hline DN & 21 & 19 & 5 \\
\hline NDRD & 9 & 7 & 1 \\
\hline TOTAL & 30 & 26 & 6 \\
\hline
\end{tabular}

Of 45 cases with DN, 21 (46.6\%) cases had DM for $<6$ yrs and $5(11 \%)$ cases had DM for $>10 \mathrm{yrs}$. Of 17 cases of NDRD, 9 (52.9\%) cases had DM for <6yrs and 1 (5.8\%) case had DM for $>10 \mathrm{yrs}$

Duration of diabetes didn't predict presence or absence of $D N$ ( $p$ value- 0.79 )

\begin{tabular}{|l|l|l|l|}
\hline CLASS OF DN & $0-5$ YEARS & 6 -10YEARS & $>$ 10YEARS \\
\hline CLASS I & 2 & 0 & 0 \\
\hline CLASS IIA & 1 & 1 & 0 \\
\hline CLASS IIB & 2 & 3 & 0 \\
\hline CLASS III & 4 & 9 & 1 \\
\hline CLASS IV & 10 & 8 & 4 \\
\hline TOTAL & 19 & 21 & 5 \\
\hline
\end{tabular}


Duration of diabetes didn't correlate with class

of DN (p value-0.53)

\section{DN Class Vs Duration of Diabetes}

-0-5YEARS 6-10YEARS ->10YEARS

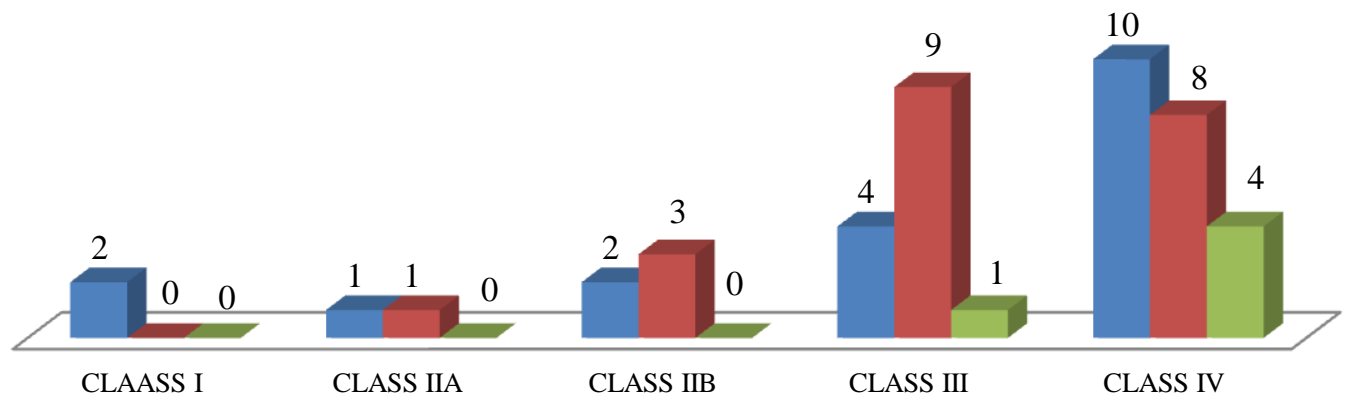

\section{Hematuria and Renal histology}

Of the 62 cases, 6 cases had microscopic hematuria. of these cases 3 had Ig A Nephropathy 2 had PIGN 1 had C3GN nephropathy .

Due to small number of cases statistical analysis could not be made.

\begin{tabular}{|l|c|c|c|}
\hline & $<3$ gms/day & $3-5$ gms/day & $>5$ gms/day \\
\hline DN & 15 & 24 & 6 \\
\hline NDRD & 10 & 5 & 2 \\
\hline TOTAL & 25 & 29 & 8 \\
\hline
\end{tabular}

Degree of protenuria didn't differentiate DN from NDRD (p value- 0.17 )

Of 17 NDRD cases, 10(58\%) had subnephrotic range protenuria and $7(41.1 \%)$ had nephrotic range

\section{DN Vs Protienuria}

Mean $24 \mathrm{hr}$ urinary protein in patients with DN was $1.98 \pm 1.85 \mathrm{~g} /$ day and mean $24 \mathrm{hr}$ urinary protein in NDRD was $1.8 \pm 1.89 \mathrm{~g} /$ day

\begin{tabular}{|l|c|c|c|}
\hline & $<3$ gms/day & $3-5 \mathrm{gms} /$ day & $>5 \mathrm{gms} /$ day \\
\hline CLASS I & 2 & 0 & 0 \\
\hline CLASS IIA & 2 & 0 & 0 \\
\hline CLASS IIB & 4 & 1 & 0 \\
\hline CLASS III & 4 & 8 & 2 \\
\hline CLASS IV & 3 & 15 & 4 \\
\hline TOTAL & 15 & 24 & 6 \\
\hline
\end{tabular}

Higher Degree of proteinuria is associated withadvanced class of DN(p value-0.03)

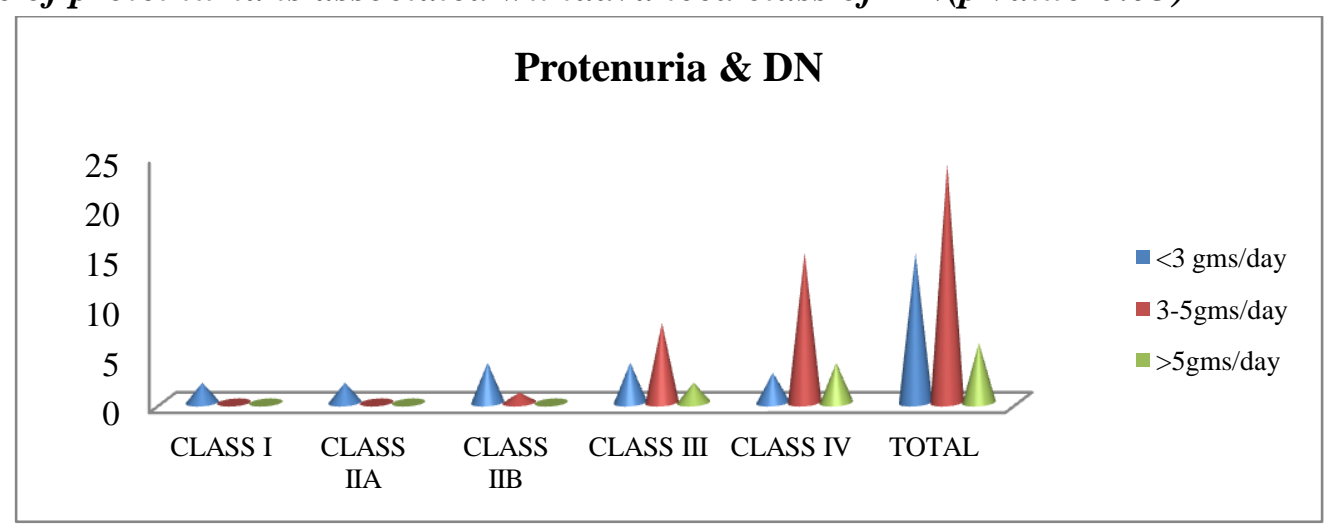




\section{DN Vs Glycemic control during admission}

$13(28.8 \%)$ of 45 patients with DN had good glycemic control vs 6(35.2\%) of 17 patients with NDRD at the time of admission.

\begin{tabular}{|l|c|c|}
\hline & Good Glycemic Control & Poor Glycemic Control \\
\hline DN & 13 & 32 \\
\hline NDRD & 6 & 11 \\
\hline TOTAL & 19 & 43 \\
\hline
\end{tabular}

Blood sugars at the time of admission poorly correlated with DN with p value-0.75

\begin{tabular}{|l|c|c|}
\hline & Good Glycemic Control & Poor Glycemic Control \\
\hline CLASS I & 0 & 2 \\
\hline CLASS IIA & 0 & 2 \\
\hline CLASS IIB & 2 & 3 \\
\hline CLASS III & 7 & 7 \\
\hline CLASS IV & 4 & 18 \\
\hline TOTAL & 13 & 32 \\
\hline
\end{tabular}

Glycemic control poorly correlated with the class of Diabetic nephropathy(p value-0.18)

DN vs. eGFR

Mean eGFR in DN was $19.69 \pm 11.69 \mathrm{ml} / \mathrm{min} / 1.732 \mathrm{~m}^{2}$ and mean eGFR of cases with NDRD was $18.4 \pm$ $11.98 \mathrm{ml} / \mathrm{min} / 1.732 \mathrm{~m}^{2}$

\begin{tabular}{|l|c|c|c|c|c|}
\hline DN/NDRD & $>90$ & $89-60$ & $59-30$ & $29-15$ & $<15$ \\
\hline DN & 0 & 1 & 5 & 21 & 18 \\
\hline NDRD & 0 & 1 & 2 & 5 & 9 \\
\hline TOTAL & 0 & 2 & 7 & 26 & 27 \\
\hline
\end{tabular}

Of 62 diabetic cases $2(3 \%)$ cases have eGFR $>60$ $\mathrm{ml} / \mathrm{min} / 1.732 \mathrm{~m}^{2}$ and $27(43.5 \%)$ cases with eGFR $<15 \mathrm{ml} / \mathrm{min} / 1.732 \mathrm{~m}^{2}$ required renal replacement therapy either in the form of hemodialysis or peritoneal dialysis.

eGFR poorly correlated with DN (p value0.75)

Correlation of baseline eGFR and histology:

Mean eGFR of cases at after stabilisation of patients (baseline GFR) in DN Class I, IIa, IIb, III and IV was $24 \pm 7.7,45.5 \pm 12.9,25.6 \pm 12.6$, $17.6 \pm 13.1, \quad 17.01 \pm 11.69 \quad \mathrm{ml} / \mathrm{min} / 1.732 \mathrm{~m}^{2}$ respectively

Higher class of DN is associated with lower eGFR i.e $50 \%$ of CLASS III and $50 \%$ of CLASS IV have $\mathrm{eGFR}<15 \mathrm{ml} / \mathrm{min} / 1.732 \mathrm{~m}^{2}$

\begin{tabular}{|l|c|c|c|c|c|}
\hline & $>90$ & $89-60$ & $59-30$ & $29-15$ & $<15$ \\
\hline CLASS I & 0 & 0 & 0 & 2 & 0 \\
\hline CLASS IIA & 0 & 1 & 0 & 1 & 0 \\
\hline CLASS IIB & 0 & 0 & 1 & 4 & 0 \\
\hline CLASS III & 0 & 0 & 1 & 6 & 7 \\
\hline CLASS IV & 0 & 0 & 2 & 9 & 11 \\
\hline TOTAL & 0 & 1 & 4 & 22 & 18 \\
\hline
\end{tabular}

Lower the eGFR higher the DN Class. (p value $<0.02)$ 


\section{Discussion}

The progressive increasing incidence of diabetic nephropathy in present population has significant implication on social and economic resources of the developing nations . Furthermore, prognosis in such patients is very poor compared with patients with ESRD due to other renal diseases. Proteinuria in diabetic patients is usually interpreted as a clinical manifestation of diabetic nephropathy. Although kidney biopsy is the most unbiased method of evaluation it is rarely used in these subjects. The primary aim of kidney biopsy in proteinuric patients with diabetic nephropathy is to confirm/exclude non-diabetic renal disease.

In 2010 Research Committee of the Renal Pathology Society (RPS) developed a consensus classification combining type 1 and type 2 diabetic nephropathies by Tervaert et al in this classification, diabetic nephropathy is divided in to four hierarchial glomerular lesions with separate evaluation for interstitial and vascular lesions. However the clinical utility of this pathological classification for predicting outcomes is not established.

This study aimed to evaluate the relationship between histological changes and clinical parameters in diabetic patients with renal dysfunction There are no standardized criteria for renal biopsy in $\mathrm{DN}$, therefore obtaining a renal biopsy from patients with DN is currently a matter of judgment by the Nephrologist. Currently, renal biopsy is commonly performed in patients with DN who show the following features-

\section{Biopsy should be considered when ${ }^{6}$}

Renal manifestations are seen atypically ( $<10$ years) early in type 1 diabetes

Dysmorphic erythrocytes/casts are found in urine (nephritic sediment)

Rapid deterioration of renal function of unknown cause is noted

Elevated serum creatinine without urine abnormalities

Macroalbuminura without retinopathy

Heavy proteinuria (>8 g/day).
Biopsy not indicated when

Typical evolution of renal disease

Concomitant retinopathy is present.

Although pathologic classifications exist for several renal diseases, a uniform classification for diabetic nephropathy is lacking. In 2010 Research Committee of the Renal Pathology Society (RPS) developed a consensus classification combining type 1 and type 2 diabetic nephropathies ${ }^{3}$

The reported incidence of NDRD ranged from 23$54 \%$ in proteinuric type 2 DM patients. ${ }^{6}$ Nondiabetic renal diseases often develop in diabetic patients with proteinuria. Therefore, kidney biopsy may become a useful diagnostic option among proteinuric patients with this type of diabetes mellitus ${ }^{3}$

With this background we analysed 62 cases of diabetes mellitus presenting with renal dysfunction (proteinuria or high serum creatinine) who underwent renal biopsy with or without classical indications of renal biopsy in diabetes and classified cases according to The Renal Pathology Society classification. In the present study of type I and Type II diabetic cases are classified together as suggested in renal pathology society classification and clinicopathological correlation was analysed.

Of the 62 cases 49 were male and 13 were females with a male to female ratio of 3.7 : 1, slightly higher in comparsion to studies by J Prakash etal ${ }^{5}$ of 1.87: 1 and 2:1 in study by Koji Harada etal. ${ }^{7}$

Mean age of cases in present study was $49.96 \pm 8.06$ years, similar to observations made by $50.68 \pm 11.29$ yrs in study by Amal Abdel Ghani etal $^{8}$

In the present study mean duration of diabetes was $6.9 \pm 4.22$ yrs. Mean duration of diabetes was $9 \pm$ 6.8 yrs in study by Sonia yaqub ${ }^{17}, 9.33 \pm 3.6 y$ yrs in study by Amal Abdel Ghani etal ${ }^{4}$ and $10.1 \pm 8.5$ years in study by Koji Harada etal ${ }^{67}$.

In the present study non diabetic renal disease (NDRD) on histopathology was present in $17(27.4 \%)$ of cases on par with Parving et al ${ }^{4}$ study which also showed that eight $(23 \%)$ of 35 type 2 diabetic patients had NDRD. Gambara et al ${ }^{10}$ 
reported that $17(32.7 \%)$ of 52 type 2 diabetic patients had NDRD. A study done by J prakash etal ${ }^{1}$ showed an incidence of NDRD in 7(30.4\%) of 23 cases of type 2 DM. Variation in incidence could due to selection bias for indication of biopsy. A meta analysis of data available on prevalence of non-diabetic kidney disease among type 2 diabetic patients done by ZukowskaSzczechowska E etal ${ }^{3}$, revealed that NDRD was evident on kidney biopsy approximately in $22 \%$ of European and $26.7 \%$ of Asian patients with type 2 diabetes mellitus similar to the present study and was observed that even after adjusting for differences in methodology among the studies, NDRD may affect a significant percentage of patients with type 2 diabetes mellitus. Therefore, kidney biopsy may become a useful diagnostic option among proteinuric patients of diabetes mellitus.

In the present study glomerular lesions were seen in $15(88.2 \%)$ cases and tubulointerstitial lesions were seen in $2(11.7 \%)$ cases. Most common NDRD in present study was IgA nephropathy seen in $4(13.5 \%)$ cases. Most common glomerular diseases were Ig A Nephropathy, Cresentric GN observed in $1(5.8 \%)$ cases each at par with the meta analysis by Zukowska-Szczechowska E $\mathrm{etal}^{3}$, in which IgA nephropathy was consistently the most common type of glomerulonephritis in both Caucasian and nonCaucasian populations accounting for approximately 6-19\% of kidney biopsies. In the study by Jianhui Zhou etal ${ }^{11}$ also Ig A nephropathy was predominant glomerular lesion accounting for $34 \%$ of cases.

In the present study mean duration of diabetes in the study population was 6.95

\pm 4.36 yrs, mean duration of diabetes in cases with biopsy proven diabetic nephropathy was $5.69 \pm 4.25$ yrs. Difference in duration of diabetes in DN and NDRD was not statistically significant with $p$ value-0.79. similar observations of no statistically significant difference in duration of DM between DN and NDRD ( $(p>0.05)$-were found in the studies by $U$. Das etal $^{12}$, Parving $\mathrm{HH}$ etal $^{4}$ and study by Amal
Abdel Ghani etal ${ }^{8}$. In a study by S. Michael Mauer etal $^{20}$, research renal biopsies were performed in patients who had Type $1 \mathrm{DM}$ for 2.5-29 yr who were selected using no other criteria there was no strong relationship between either glomerular basement membrane (GBM) thickness or mesangial expansion and duration of Type $1 \mathrm{DM}$. In the present study mean duration of DM in DN Class I, II a, II b, III and IV was $4 \pm 2.3,5 \pm 4.5$, $4.6 \pm 3.6,7.7 \pm 3.9$ and $7.4 \pm 4.2$ yrs respectively. There was no statistically significant difference in duration of diabetes and class of $D N$ with $p$ 0.523. Melvin M. Schwatrz etal ${ }^{13}$ noted that there was significant difference in duration of diabetes between patients with K-W lesions and mesangial lesions.

Of the 62 cases, 32 pts $(51.6 \%)$ had diabetic retinopathy, of which 23 cases $(71.8 \%)$ had $\mathrm{DN}$, 9cases $(28.1 \%)$ had NDRD. In study of renal biopsy in patients with presumed diabetic nephropathy by Koji Harada etal ${ }^{7}$ of the 21 cases with DR 18 (85.7\%) had DN, J Prakash ${ }^{5}$ reported $9(60 \%)$ cases with retinopathy had DN and $40 \%$ had NDRD.

Of 30 cases who did not have diabetic retinopathy 22 cases $(73.3 \%)$ had biopsy proven DN on par with a study done by Perk. Christensen etal of 52 patients with type 2 diabetes without diabetic retinopathy, 35(69\%) patients had diabetic nephropathy on biopsy, Serra et $\mathrm{al}^{14}$ reported that diabetic glomerulosclerosis was diagnosed in $17(74 \%)$ of patients without diabetic retinopathy. Schwartz MM etal ${ }^{13}$ noted 7 of $8(87.5 \%)$ patients without retionopathy had mesangiosclerosis characteristic of DN, J prakash etal ${ }^{5}$ noted that 4 of $8(50 \%)$ cases without DR had DN. It should be pointed out that absence of retinopathy cannot exclude the presence of diabetic nephropathy.

Clearly diabetic nephropathy can occur in absence of retinopathy in Type 2 proteinuric diabetic patients. (p value-0.8)

Type 2 diabetic patients with typical diabetic nephropathy on biopsy did not have diabetic retinopathy in the study by $\mathrm{J}$ prakash etal ${ }^{5}$, Parving et $\mathrm{al}^{4}$. noted that $40 \%$ of proteinuric Type 
2 DM patients lacked diabetic retinopathy.Of 20 cases of NDRD $8(40 \%)$ cases had diabetic retinopathy, in study by Prakash J etal. ${ }^{75}$

Management of cases with NDRD grossly differs from that of $D N$, if biopsy is not done in patients with DR presuming diagnosis of DN significant number of NDRD requiring specific therapy might be missed.

Presence or absence of Diabetic retinopathy poorly correlated with presence or absence of diabetic nephropathy $p$ value $\mathbf{- 0 . 8}$

Thus renal biopsy is necessary for precise diagnosis of diabetic and non-diabetic renal lesions in proteinuric Type 2 diabetic patients even in the presence of diabetic retinopathy.

In the present study both cases of Class I DN, Class IIa did not have DR, $20 \%$ of cases of Class IIb DN had DR, 50\% of Class III DN had DR and $68.8 \%$ of cases with Class IV DN had DR.

Prescence of diabetic retinopathy correlated with higher class of diabetic

nephropathy ( pvalue-0.03), similar observations were noted by Koji Harada etal ${ }^{15}$ patients with both DN and DR showed more severe renal histology than those without DR. Melvin M. Schwatrz etal ${ }^{13}$ noted that patient with $\mathrm{K}-\mathrm{W}$ lesions had correlation with retinopathy but not mesangial sclerosis, similar observations were made in type $1 \mathrm{DM}$ also- by Blanchem. Chavers etal. $^{16}$

Mean $24 \mathrm{hr}$ urinary protein in patients with DN was $1.98 \pm 1.85 \mathrm{~g} /$ day and mean $24 \mathrm{hr}$ urinary protein in NDRD was $1.8 \pm 1.89 \mathrm{~g} /$ day. Degree of protenuria didn't differentiate $D N$ from NDRD (p value-0.17)

Mean 24hr urinary protein in DN Class I, IIa, IIb, III and IV was $0.68 \pm 0.96,0.7 \pm 2.06,1.66 \pm 1.84$, $2.2+1.85$ and $2.14+1.85$ g/day respectively. Degree of protenuria correlated with class of $D N$ (p-value-0.03), similar observations were made by Kathryn Elizabeth White $^{18}$, RuthoSterbyetal ${ }^{19}$ where severity of proteinuria correlated with index of structural lesions.

$13(28 \%)$ of 45 cases of DN had poor glycemic control. 6 (35\%) of 17 cases of NDRD had poor glycemic control. Glycemic control at time of biopsy could not predict DN or NDRD p value 0.75. Similar observations were made by parving etal. ${ }^{4}$ with a $\mathrm{p}$ value $>0.5$.

Glycemic control poorly correlated with class of diabetic nephropathy $p$ value -0.18. Melvin $\mathrm{M}$. Schwatrz etal ${ }^{13}$ noted that glycemic control correlated poorly with patients with $\mathrm{KW}$ lesions and mesangial sclerosis..

Of the 45 cases with DN, $34(75.5 \%)$ cases had hypertension. and $11(24.4 \%)$ cases have normotension. Of 17 cases with NDRD 12(70.5\%) were hypertensive.

Hypertension did not differentiate between DN and NDRD (p value-0.94). similar observations were made by Amal Abdel Ghani etal ${ }^{5}$, Vincenzo Gambara etal $^{10}$, Sonia yaqub etal ${ }^{17}$.

$77.2 \%$ of cases with Class IV DN had HTN. Presence of hypertension did not correlate with class of Diabetic nephropathy ( $p$ value-0.1)

Mean eGFR in DN was $19.69 \pm 11.69$ $\mathrm{ml} / \mathrm{min} / 1.732 \mathrm{~m}^{2}$ and mean eGFR of cases with NDRD was $18.4 \pm 11.98 \mathrm{ml} / \mathrm{min} / 1.732 \mathrm{~m}^{2}$. Higher class of DN is associated with lower eGFR i.e $50 \%$ of CLASS III and $50 \%$ of CLASS IV have eGFR $<15 \mathrm{ml} / \mathrm{min} / 1.732 \mathrm{~m}^{2}$

mean eGFR didn't differentiate between $D N$ and NDRD (p value-0.75)

Mean eGFR of cases at after stabilisation of patients (baseline GFR) in DN Class I, IIa, IIb, III and IV was $24 \pm 7.7,45.5 \pm 12.9,25$. $6 \pm 12.6$, $17.6 \pm 13.1, \quad 17.01 \pm 11.69 \quad \mathrm{ml} / \mathrm{min} / 1.732 \mathrm{~m}^{2}$ respectively, with a significant difference between DN classes $p$ value $<0.020$. Lower the $\boldsymbol{e}$ GFR higher the DN Class (p value-0.02). Melvin M. Schwatrz et al noted that there was significant difference in creatinine clearance between patients with $\mathrm{K}-\mathrm{W}$ lesions and mesangial lesions.

\section{Conclusions}

1) Non diabetic renal disease constitutes significant percentage of patients with clinical diabetic nephropathy patients and its presence is highly underestimated. 
2) Ig $\mathrm{A}$ is most common histopathological lesions among NDRD patients.

3) The most common histopathological lesion was diabetic nephropathy among all diabetic patients with renal dysfunction.

4) Majority of patients with diabetic nephropathy are CLASS IV.

5) Higher degree of proteinuria, presence of diabetic retinopathy ,low eGFR hypertension correlated with higher classes of diabetic nephropathy .

6) Degree of proteinuria ,duration of diabetes, diabetic retinopathy and glycemic control, poorly predicted the occurrence of NDRD.

7) Renal biopsy is the unbiased method for precise diagnosis of diabetic and non diabetic renal lesions.

\section{References}

1. Kramer CK, Leitao CB, Pinto LC, Silveiro SP, Gross JL, Canani LH: Clinical and laboratory profile of patients with type 2 diabetes with low glomerular filtration rate and normoalbuminuria. Diabetes Care 2007, 30:1998-2000.

2. Fioretto P, Steffes MW, Sutherland DE, Goetz FC, Mauer M: Reversal of lesions of diabetic nephropathy after pancreas transplantation. $N$ Engl J Med 1998, 339:69-75.

3. Zukowska-Szczechowska E, Tomaszewski M. Renal affection in patients with diabetes mellitus is not always caused by diabetic nephropathy. Annales Academiae medicae Bialostocensis 2004;49:185-189

4. Parving HH, Gall MA, Skott P, Jorgensen $\mathrm{HE}$, et al. Prevalence and causes of albuminuria in non-insulin-dependent diabetic patients. Kidney Int 1992;41:75862.

5. J Prakash, $M$ Lodha, SK Singh, et al.Diabetic Retinopathy is A Poor Predictor of Type of Nephropathy in Proteinuric Type 2 Diabetic Patients. JAPI JUNE 2007; 55 :412-416
6. Jai prakash Non-Diabetic Renal Disease (NDRD) in Patients with Type 2 Diabetes Mellitus (Type 2 DM). JAPI 2013; 61:194-199

7. Koji Harada etal.. Significance of renal biopsy in patients with presumed diabetic nephropathy. Journal of Diabetes Investigation 2013; 4 (1), p88-93.

8. Amal Abdel Ghani etal.. Renal biopsy in patients with type 2 diabetes mellitus: indic ations and nature of lesions. Ann Saudi Med 2009;29(6):450-453.

9. S. Michael Mauer, Michael W. Steffes etal.. Structural-Functional Relationships in Diabetic Nephropathy. J. Clin. Invest. 1984; 74: 1143-1155.

10. Gambara V, Mecca G, Remuzzi G, Bertani $\mathrm{T}$. Heterogeneous nature of renal lesions in type II diabetes. J Am Soc Nephrol 1993;3: 1458-1466.

11. Jianhui Zhou etal..A differential diagnostic model of diabetic nephropathy and nondiabetic renal diseases. Nephrol Dial Transplant 2008; 23: 1940-1945.

12. U.Das, Dakshinamurthy, etal..Non diabetic renal disease in type 2 diabetic patients: Single centre experience. Indian Journal of nephrology 2012;22(5): 358-362

13. Schwartz MM etal. Renal pathology patterns in type II diabetes mellitus: relationship with retinopathy. Nephrol Dial Trasnsplant 1998;13:2547-2552.

14. Serra A, Romero R, Bayes B et al. Is there a need for changes in renal biopsy criteria in proteinuria in Type 2 diabetes? Diabetes Research and Clinical Practice 2002;58:149-53.

15. Prakash J etal.. Non-Diabetic Renal Disease in Patients with Type 2 Diabetes Mellitus J Assoc Physicians India. 2001;49:415-20.

16. Blanchem. Chavers, S. Michaelmauer etal. Relationship between retinal and Glomerular lesions in IDDM patients. DIABETES 1994;43:p 441-446. 


\title{
JMSCR Vol||05||Issue||11||Page 30002-30014||November
}

17. Sonia yaqub etal..Non-Diabetic renal disease in in patients with type 2 diabetes mellitus. Saudi J Kidney Dis Tanspl 2012;23(5): 1000-1007.

18. . Kathryn Elizabeth White and Rudolf William Bilous etal.. Type 2 Diabetic Patients with Nephropathy Show Structural- Functional Relationships that Are Similar to Type 1 Disease. J Am Soc Nephrol 2000;11: 1667-1673.

19. Ruth Osterby, Hans-Henrikparving, Glomerular Structure and Function in Diabetic Nephropathy Early to Advanced Stages. DIABETES, 1990; 39:1057-1063

\author{
Abbreviations \\ DM - Diabetes Mellitis \\ HTN - Hypertension \\ PDR- Proliferative Diabetic Retinopathy \\ NPDR- Non Proliferative Diabetic Retinopathy \\ CSME- Clinically Significant Macular Edema \\ HEP B-Hepatitis B \\ HEP C-Hepatitis C \\ PN-Peripheral Neuropathy \\ CVA-Cerebro Vascular Accident \\ CAD-Coronary Artery Disease \\ eGFR-Estimated Glomerular Filtration Ratio \\ DN-Diabetic Nephropathy \\ NDRD-Non Diabetic Renal Disease \\ C3GN-C3 Glomerulonephritis \\ ATIN-Acute Tubulointerstial Nephropathy \\ CIN-Chronic Interstitial Nephritis \\ PIGN-POST Infectious Glomerulonephritis
}

